\title{
Comparison of DNA probe (Gen-Probe) with culture for the detection of Neisseria gonorrhoeae in an urban STD programme
}

\author{
J R Schwebke, M E Zajackowski
}

Background and Objectives: The DNA probe assay is an alternative to culture for the detection of Neisseria ganorrhoeae which does not depend on the viability of the organism. There have been few published studies comparing the two methodologies. The majority of these studies have shown the probe assay to be comparable to culture. The goal of this study was to compare the performance of the DNA probe assay with culture in a high prevalence setting with nearly optimal culture transport conditions.

Methods: Genital specimens for culture and for DNA probe assay were collected at the time of the routine visit for STD clinic patients. Results of the two methods were compared. Discrepant results were further analysed using the results of direct patient Gram stains.

Results: Satisfactory matched specimens were available for 999 patients. The overall sensitivity and specificity of the DNA probe as compared with culture was 90 and $96 \%$. Resolved values achieved by factoring in the results of direct patient Gram stains did not significantly improve the performance of the probe.

Conclusion: Culture remains the test of choice for the detection of gonorrhoea in a high prevalence setting where culture transport conditions are adequate.

(Genitourin Med 1996;72:108-110)

Keywords: Neisseria gonorrhoeae; DNA probe; culture

\section{Introduction}

Currently, with the exception of Gram stain, the only available testing method alternative to culture for the detection of Neisseria gonorrhoeae is the unamplified DNA probe (GenProbe PACE 2, Gen-Probe Inc, San Diego, CA). The potential advantage of the probe assay over culture is its ability to detect nonviable as well as viable organisms. The relative fastidious nature of the gonococcus may lead to loss of viability, and therefore false negative cultures, in instances where cultures are not properly incubated or must be shipped long distances to the laboratory. ${ }^{1-4}$ The growth of $N$ gonorrhoeae may also be affected by recent use of antibiotics by patients, a common phenomenon among patients attending sexually transmitted disease (STD) clinics. ${ }^{5}$

Finally, DNA probe testing more easily lends itself to semi-automation and thus may result in cost-savings for personnel. These perceived advantages have led some STD control programmes to substitute this methodology for culture for the detection of gonorrhoea, despite the fact that there have been relatively few published studies of the performance of the assay. ${ }^{6-11}$ Prior to serious consideration of implementing the DNA probe as the primary testing modality for gonorrhoea in its STD clinics, the Chicago Department of Health compared the assay with culture in a setting where transport conditions to the central laboratory were adequate.

\section{Methods}

Study population

The study was conducted at each of the five Chicago Department of Health clinics scat- tered throughout the city which specialised in the diagnosis and treatment of STDs. During the month of January, 1994, all patients seen for a new problem were screened for gonorrhoea with both culture and DNA probe assays. The usual prevalence of gonorrhoea in this population as determined by the clinic's activity reports was estimated to be $10 \%$ for women and $21 \%$ for men.

\section{Specimen collection}

Endocervical specimens were obtained by inserting an unlubricated speculum into the vagina and exposing the cervix. Following cleaning of the ectocervix with a large swab, a cotton-tipped swab was inserted into the os and gently rotated. The specimen was first placed onto a clean glass slide for Gram's stain and then inoculated onto a modified ThayerMartin agar plate at the bedside. Urethral specimens were obtained by inserting a small diameter calcium alginate swab $2-3 \mathrm{~cm}$ into the urethra. Following gentle rotation, the swab was withdrawn and a slide and agar plate prepared as above. Specimens for DNA probe testing were collected following the specimens for culture in the same manner, using the swab provided in the test kits.

\section{Specimen transport}

Culture plates were placed into candle jars within 30 minutes of inoculation. Jars were incubated at $35^{\circ} \mathrm{C}$ within one hour. Plates were incubated for $24-48$ hours at the clinics prior to transportation to the Illinois Department of Public Health Laboratory's Chicago location. DNA probe specimens were refrigerated for transport to the laboratory on the next business day. All specimens were 
shuttled to the laboratory by couriers as part of their daily route.

\section{Specimen processing}

Slides prepared directly from clinical specimens were Gram stained and examined by light microscopy at $100 \times$ magnification for the presence of intracellular Gram negative diplococci typical of $N$ gonorrhoeae. Cultures for $N$ gonorrhoeae were incubated at $35^{\circ} \mathrm{C}$ in $3-5 \% \mathrm{CO}_{2}$ for a total of 48 hours prior to examination. The presence of $N$ gonorrhoeae was determined by presumptive identification using growth on Thayer-Martin agar, Gram stain morphology and positive oxidase reaction as criteria. ${ }^{12}$ DNA probe assays were performed in exact accordance with the manufacturer's instructions. The assay detects ribosomal RNA by means of a chemiluminescent labelled DNA probe. Specimens were determined to be positive if their chemiluminescence response was $\geqslant 300$ relative light units (RLUs) more than the response of the negative reference control.

\section{Results}

Specimens for culture and DNA probe were collected simultaneously from 1009 patients during the month of January, 1994. Six patients were subsequently unevaluable for inclusion owing to unsatisfactory cultures, and four were eliminated because of unsatisfactory DNA probe specimens (inadequate patient identification) leaving 999 evaluable patients. Of these, $453(45 \%)$ were female and 546 $(55 \%)$ were male. Cultures were positive in $78 / 453(17 \%)$ of the females and $153 / 546$ $(28 \%)$ of the males. Disparate results occurred among 55 specimen pairs. Using culture as the gold standard, there were 31 false positive and 24 false negative DNA probe assays. The sensitivity, specificity, and positive and negative predictive values of the probe assay are shown in the table.

Medical records were identified for all but one of the patients with discrepant results. Of the males with false negative probe results, $13 / 13(100 \%)$ complained of symptoms versus $9 / 17(53 \%)$ of males with false positive probe results. Urethral discharge was present on examination in $12 / 13(92.3 \%)$ of males with culture positive, probe negative specimens versus $5 / 17(29.4 \%)$ of males with culture negative, probe positive specimens.

Further analysis of the patients in the false positive DNA probe group revealed that 5 patients ( 3 male and 2 female) had positive direct Gram stains for gonorrhoea at the time of their visit. Of these 5 patients, 4 also had RLUs of $>10,000$ suggesting that these patients may have had false negative cultures. Assuming that these 5 patients were truly infected, the resolved overall sensitivity and specificity for the DNA probe was 89.8 and $96.6 \%$ respectively. The resolved sensitivity and specificity of the test when performed in males was 91.7 and $96.4 \%$ and in females, 86.2 and $96.8 \%$. These resolved values did not differ significantly from the original values.

Only three patients with discrepant results (all male) admitted to the recent use of antibiotics. Two of these were among the false positive probe patients and both had RLUs of $<1000$.

\section{Discussion}

An important component for the control of any STD is a diagnostic test which is affordable, technically simple and which has a high degree of sensitivity and specificity. For many years, culture for $N$ gonorrhoeae on selective media has met those specifications. Culture also has the advantages of good performance for the isolation of $N$ gonorrhoeae from nongenital sites and has the ability to provide viable organisms for the monitoring of susceptibility trends.

Admittedly, culture techniques for gonorrhoea do have some limitations. ${ }^{1-4}$ The ability of the organism to grow on the agar plate may be affected by adverse environmental factors such as inappropriate incubation temperature or level of $\mathrm{CO}_{2}$. Some strains of $\mathrm{N}$ gonorrhoeae may be susceptible to vancomycin, one of the inhibitory antibiotics contained in the selective media. ${ }^{13}$ Lastly, the patient may have recently ingested antibiotics which may inhibit growth of the organism. Despite these limitations, the sensitivity of culture has been generally regarded to be between $80-95 \% .{ }^{14} \mathrm{~A}$ recent study by Smith et al found the sensitivity of culture to be $96.4 \%$ when compared with newly developed amplified antigen detection techniques. ${ }^{15}$

Owing to the recognition of these limitations, particularly those that relate to transport conditions in the field, some STD programmes have begun to utilise the DNA probe instead of culture. Previously published studies, although limited in number, have suggested that the DNA probe assay is equivalent to culture for the detection of $N$ gonorrhoeae. Four of these studies reported sensitivities ranging from $92-100 \%$ with specificities of 91-99\%.7 81011 Panke et al documented an initial sensitivity of $88 \%$ for endocervical

Sensitivity, specificity, and predictive value of DNA probe compared with culture

\begin{tabular}{|c|c|c|c|c|}
\hline & Sensitivity (\%) & Specificity (\%) & $P V P(\%)^{\star}$ & $P V N(\%)+$ \\
\hline $\begin{array}{l}\text { Total } \\
(\mathrm{n}=999,231 \text { culture positive, } 207 \text { probe positive }) \\
\text { Male urethral }\end{array}$ & $89 \cdot 6$ & $96 \cdot 0$ & $87 \cdot 0$ & $96 \cdot 8$ \\
\hline $\begin{array}{l}(\mathrm{n}=546,153 \text { culture positive, } 140 \text { probe positive }) \\
\text { Endocervical }\end{array}$ & $91 \cdot 5$ & $95 \cdot 7$ & $89 \cdot 2$ & $96 \cdot 6$ \\
\hline ( $n=453,78$ culture positive, 67 probe positive) & $85 \cdot 9$ & $96 \cdot 3$ & $82 \cdot 7$ & $97 \cdot 0$ \\
\hline
\end{tabular}

^Predictive value of a positive test. 
specimens but subsequently concluded the assay was as sensitive as culture when a modification of the manufacturer's cut-off value was utilised. ${ }^{6}$ The only published study which has compared probe with culture under conditions that included long distance transport, utilised specimens obtained from family planning clinics that had a $1 \%$ prevalence of gonorrhoea. This study also altered the manufacturer's cutoff value by classifying those specimens with RLUs in the "grey-zone" (300-500) as suspicious. When suspicious specimens were included as positives, the sensitivity and specificity in this low prevalence population were both $98 \%$; however, the positive predictive value of the probe assay was only $37 \cdot 6 \% .{ }^{9}$

Our findings differ from those of previously reported studies, with the exception of Panke et al, in that we found the overall sensitivity of the probe assay to be less than $90 \%$. In women, the sensitivity fell to $86 \%$. Use of the direct patient Gram stain to resolve discrepant results failed to significantly improve the sensitivity, although we acknowledge that endocervical Gram stains for the detection of gonorrhoea have limited sensitivity and thus some of the false positive probe specimens in females may have continued to be misclassified.

Of concern also is the relatively poor specificity of the DNA probe assay in this study. After adjusting for available Gram stain data, $26 / 999(2.6 \%)$ of patients had false positive probes. Such problems with specificity would be magnified in lower prevalence populations. It is possible that prior antibiotic usage by our patients may have influenced specificity by inhibiting growth of organisms in culture, yet allowing for the detection of ribosomal RNA from non-viable organisms.

Although only $3 / 55(5.5 \%)$ of patients with discrepant test results admitted to antibiotic usage, studies of antibiotic usage patterns among STD clinic patients suggest that the actual number of such patients may have been greater. ${ }^{516}$ Interestingly, however, Reichart et al found no significant differences in gonorrhoea culture positivity rates when comparing patients who had recently taken antibiotics with those who had not. ${ }^{5}$

A potential limitation of our study was the use of presumptive as opposed to confirmative diagnosis of $N$ gonorrhoeae. Although it is true that $N$ meningitidis can be isolated from the urogenital tract, in the majority of cases it is the anal canal which is involved. Meningococci are rarely isolated from the endocervix of women or the urethra of heterosexual males. In a study by Smeltzer et al, $N$ meningitidis was isolated from only $2 / 25,936$ of endocervical specimens. ${ }^{12}$ Carlson et al found $N$ menigitidis in $0.2 \%$ of urethral cultures obtained from heterosexual males. ${ }^{17}$ Thus, although it is possible that a presumptively identified isolate in our study was misclassified as $N$ gonorrhoeae, the chances are small.

In summary, in a high prevalence setting, the DNA probe assay did not compare favourably with culture, and in fact if used in our setting would fail to detect over $10 \%$ of cases. We conclude that in high prevalence settings with easy access to the diagnostic laboratory, culture remains the gold standard for the detection of $N$ gonorrhoeae. The use of DNA probe assays in these settings may negatively impact disease control measures.

We acknowledge the assistance of the microbiologists at the Illinois Department of Public Health Laboratory, and the STD/HIV Program Staff. We thank Gene Jakubowski and Charity Richey for data management support and Windell Ross for her expert assistance with manuscript preparation.

1 Bonin P, Tanino TT, Handsfield HH. Isolation of Neisseria gonorrhoeae on selective and nonselective media in a sexually transmitted disease clinic. F Clin Microbiol 1984; 19:218-20.

2 Goodhart ME, Ogden J, Zaidi AA, Kraus SJ. Factors affecting the performance of smear and culture tests for detection of Neisseria gonorrhoeae. Sex Transm Dis 1982; 9:63-9.

3 Hook EW III, Handsfield $\mathrm{HH}$. Gonococcal infections in the adult. In: Holmes KK, Mardh PA, Sparling PG et al,
eds. Sexually Transmitted Diseases. New York: McGraweds. Sexually Transmi

4 Sng EH, Rajan VS, Yeo KL, Goh AJ. The recovery of Neisseria gonorrhoeae from clinical specimens: effects of different temperatures, transport times, and media. Sex Transm Dis 1982;9:74-8.

5 Reichart CA, Neumann T, Foreman P, Zenilman JM, Hook EW III. Temporal trends in gonococcal antibiotic resistance in Baltimore. Sex Transm Dis 1992;19:213-8.

6 Panke ES, Yang LI, Leist PA, Magevney P, Fry RI, Lee RF Coris ture for the detection of Neisseria gonorrhoeae in genital ture for the detection of Neisseria gon
samples. Sex Trans Dis 1993;20:243-7.

7 Vlaspolder F, Mutsaers JAEM, Blog F, Notowicz A. Value of a DNA probe assay (Gen-Probe) compared with that of culture for diagnosis of gonococcal infection. $₹ \mathrm{Clin}$ Microbiol 1993;31:107-10

8 Stary A, Kopp W, Zahel B, Nerad S, Teodorowicz L, Horting-Muller I. Comparison of DNA-probe test and culture for the detection of Neisseria gonorrhoeae in genital samples. Sex Transm Dis 1993;20:243-7.

9 Limberger RJ, Biega R, Evancoe A, McCarthy L, Slivienski L, Kirkwood M. Evaluation of culture and the GenProbe PACE 2 assay for detection of Neisseria gonorrhoeae and Chlamydia trachomatis in endocervical specimens and Chlamydia thach 1992;30:1162-6.

10 Granato PA, Franz MR. Evaluation of a prototype DNA probe test for the non-cultural diagnosis of gonorrhea. $\mathcal{F}$ Clin Microbiol 89;27:632-5.

11 Hale YM, Melton ME, Lewis JS, Willis DE. Evaluation of the PACE 2 Neisseria gonorrhoeae assay by three public health laboratories. $\mathcal{F}$ Clin Microbiol 1993;31:451-3.

12 Smeltzer MP, Curran JW, Brown ST, Pass J. Accuracy of presumptive criteria for culture diagnosis of Neisseria gonorrhoeae in low-prevalence populations of women. $7 \mathrm{Clin}$ Microbiol 1980;11:485-7.

13 Windall JJ, Hall MM, Washington JA II. Inhibitory effects of vancomycin on Neisseria gonorrhoeae in Thayer-Martin medium. $\mathcal{Y}$ Infect Dis $1980 ; 142: 775$.

14 Hook EW III, Hansfield HH. Gonococcal infections in the adult. In: Holmes KK, Mardh PA, Sparling PG et al, eds. adult. In: Holmes KK, Mardh PA, Sparling PG et al, eds. 1990:149-65.

15 Smith KR, Ching S, Lee $\mathrm{H}$, et al. Evaluation of ligase chain reaction for use with urine for identification of Neisseria gonorrhoeae in females attending a sexually transmitted disease clinic. F Clin Microbiol 1995;33:455-7.

16 Gordon SM, Mosure DJ, Lewis J, Brown S, McNagny SE, Schmid GP. Prevalence of self-medication with antibiotics among patients attending a clinic for treatment of sexually transmitted diseases. Clin Infect Dis 1993;17: 462-5.

17 Carlson BL, Fiumara NJ, Kelly JR, McCormack WM. Isolation of Neisseria menigitidis from anogenital specimens from homosexual men. Sex Transm Dis 1980;7: 71-3. 